\title{
An immune response after intraocular administration of an adenoviral vector containing a $\beta$ galactosidase reporter gene slows retinal degeneration in the $r d$ mouse
}

\author{
M B Reichel, J Bainbridge, D Baker, A J Thrasher, S S Bhattacharya, R R Ali
}

University Eye

Hospital, Liebigstrasse 10-14, D-04103 Leipzig, Germany M B Reichel

Department of Molecular Genetics, Institute of

Ophthalmology, UCL, London EC1V 9EL, UK M B Reichel

J Bainbridge

S S Bhattacharya

R R Ali

Neuroinflammation Group, Institute of Neurology, UCL, London WC1N 1PJ

D Baker

Molecular

Immunology Unit, Institute of Child Health, UCL, London

WC1N 1EH

A J Thrasher

R R Ali

Correspondence: Dr Robin Ali, Department of Molecular Genetics, Institute of Ophthalmology, UCL, Bath Street, London EC1V 9EL, UK

r.ali@ucl.ac.uk

Accepted for publication 13 September 2000

\begin{abstract}
Backgroundlaims-Retinal degenerations are a leading cause of blindness for which there are currently no effective treatments. This has stimulated interest in the investigation of gene therapy strategies for these diseases in a variety of animal models. A number of attempts have been made to prevent photoreceptor loss in the rd mouse model of retinal degeneration using adenoviral vectors containing either a copy of the missing functional gene or a gene encoding either a neurotrophic factor or an antiapoptotic factor. The authors have previously demonstrated that intraocular administration of an adenoviral vector containing a $\beta$ galactosidase gene (AV.LacZ) results in an immune response to viral gene products and $\beta$ galactosidase. Here the effect of the immune response on retinal degeneration is examined.

Methods-Juvenile $r d$ mice were injected intravitreally with AV.LacZ and a proportion were depleted of either CD4+ or CD8+ $T$ cells or both. Control animals were injected with PBS. The mice were sacrificed 10 and 20 days post-injection and their eyes embedded in paraffin wax and sectioned.

Results-10 days after intravitreal injection of AV.LacZ, the outer nuclear layer contains an average of 2.5 rows compared with 1.5 in PBS injected animals $(p<0.005)$. The protective effect of AV.LacZ is negated by immune suppression and does not extend beyond 20 days. Conclusion-An immune response to vector and transgene products is able to slow degeneration in the $r d$ mouse. This phenomenon should be taken into account when analysing the degeneration in the $r d$ mouse following gene transfer.

(Br f Ophthalmol 2001;85:341-344)
\end{abstract}

Retinal degeneration is a common cause of blindness for which there is still no treatment. Of all the retinal degenerations, retinitis pigmentosa (RP) is the best characterised. ${ }^{1}$ It has an overall prevalence of up to 1 in 3000 and leads to progressive loss of vision, initially manifesting as night blindness and reduction of the peripheral visual field, and later involving central vision. RP is clinically and genetically heterogeneous with 28 mapped loci and 11 identified genes, seven of which are expressed exclusively in photoreceptor cells. ${ }^{2-8} \mathrm{~A}$ number of well characterised animal models with gene defects analogous to those found in humans have increased our understanding of these diseases and also provide models to test treatment strategies. The retinal degeneration $(r d)$ mouse is perhaps the most extensively investigated animal model for RP. ${ }^{9}$ It is homozygous for a spontaneously occurring null mutation in the gene which encodes the $\beta$ subunit of rod specific cyclic GMP phosphodiesterase $(\beta \mathrm{PDE})$. Analogous mutations in the gene encoding $\beta$ PDE in humans results in autosomal recessive RP. The lack of functional PDE in the $r d$ mouse results in a rapid and severe degeneration. Photoreceptors are lost within 1 week of birth, with $50 \%$ loss by 2 weeks and almost complete loss by 4 weeks. As in other models of retinal degeneration, it has been demonstrated that photoreceptors are lost through an apoptotic mechanism..$^{10}$ Heterozygous $r d$ mice are phenotypically indistinguishable from wild type animals.

Three different gene therapy strategies have been followed in attempts to slow the degeneration in the $r d$ mouse-introduction of a functional copy of the $\beta$ PDE gene to photoreceptor cells in order to test the efficacy of gene replacement therapy ${ }^{11-13}$; introduction of additional copies of the $b c l-2$ gene to photoreceptor cells in order to block apoptosis directly ${ }^{14}$; and, finally, introduction of genes encoding secreted neurotrophic factors to cells in the anterior segment of the eye $\mathrm{e}^{15}$ in order to support degenerating photoreceptors by indirectly blocking apoptosis. The latter two strategies while not capable of improving vision may help maintain it by preserving rods and therefore indirectly preserving cones which do not have the rod specific enzyme defect but are nevertheless eventually lost. In vitro ${ }^{16}$ and in vivo ${ }^{17}$ data suggest that diffusible trophic factors released by rods promote cone cell survival.

Adenoviral vectors (AV) have been widely used for gene transfer to the eye and there have been a number of reports of delayed retinal degeneration in $r d$ mice following subretinal delivery of $\mathrm{AV}$ and $\mathrm{AV}$ encapsulated vectors carrying a gene encoding either $\beta \mathrm{PDE}^{11}{ }^{13}$ or $\mathrm{Bcl}-2^{14}$ or intravitreal delivery of an $\mathrm{AV}$ vector carrying a gene encoding ciliary neurotrophic factor (CNTF). ${ }^{15}$ We have previously demonstrated that intraocular administration of an adenoviral vector containing a $\beta$ galactosidase (lacZ) gene results in retinal inflammation and a $\mathrm{T}$ cell mediated immune response to viral 
and $\beta$ galactosidase products. ${ }^{18}$ Furthermore, immune suppression by administration of $\mathrm{T}$ cell specific monoclonal antibodies prolonged the duration of lacZ gene expression. In order to determine the effects of an immune response to adenoviral vector and transgene products on retinal degeneration, we intravitreally injected $r d$ mice with an $\mathrm{AV}$ vector carrying a lacZ reporter gene with and without immune suppression. Our results suggest that the immune response itself is able to slow degeneration in the $r d$ mouse.

\section{Materials and methods}

ANIMALS AND INJECTIONS

The recombinant vector used was an E1 deleted recombinant human 5 a adenoviral vector, $\mathrm{pXCXRBb}$, containing a lac $\mathrm{Z}$ reporter gene driven by a rous sarcoma virus LTR promoter (AV.LacZ). This was a kind gift from Dr A P Byrnes, Department of Molecular Microbiology and Immunology, Johns Hopkins University School of Public Health, USA and has been previously described. ${ }^{19}$ Intravitreal injections were carried out in 12 day old $r d$ mice $(\mathrm{C} 3 \mathrm{H}$, Harlan Olac Ltd, UK). Mice were anaesthetised by intraperitoneal injection of $0.2 \mathrm{ml}$ Hypnorm (Janssen Pharmaceutical Ltd, Oxford), and Hypnovel (Roche, Welwyn Garden City) mixed 1:1:6 with distilled water. The eyes were protruded by gentle pressure on the animal's mandible. Once the eye had been proptosed, it was held in position by a rubber sleeve which was placed around the eye with a pair of forceps. The pressure of the rubber sleeve on the eye was always moderate and did not block the circulation. The eye was additionally stabilised by holding an extraocular muscle with a pair of fine forceps. The tip of a $1.5 \mathrm{~cm}, 34$ gauge needle (Hamilton, Switz) was tunnelled transclerally through the pars plana into the vitreous, in parallel with the limbus. Either $1 \mu \mathrm{l}$ of phosphate buffered saline (PBS) or $1 \mu \mathrm{l}$ of Av.LacZ $\left(1 \times 10^{6} \mathrm{pfu}\right)$ was injected. Only one eye per animal was processed. Some of the animals were also depleted of $\mathrm{T}$ cells by intraperitoneal injection at of 250

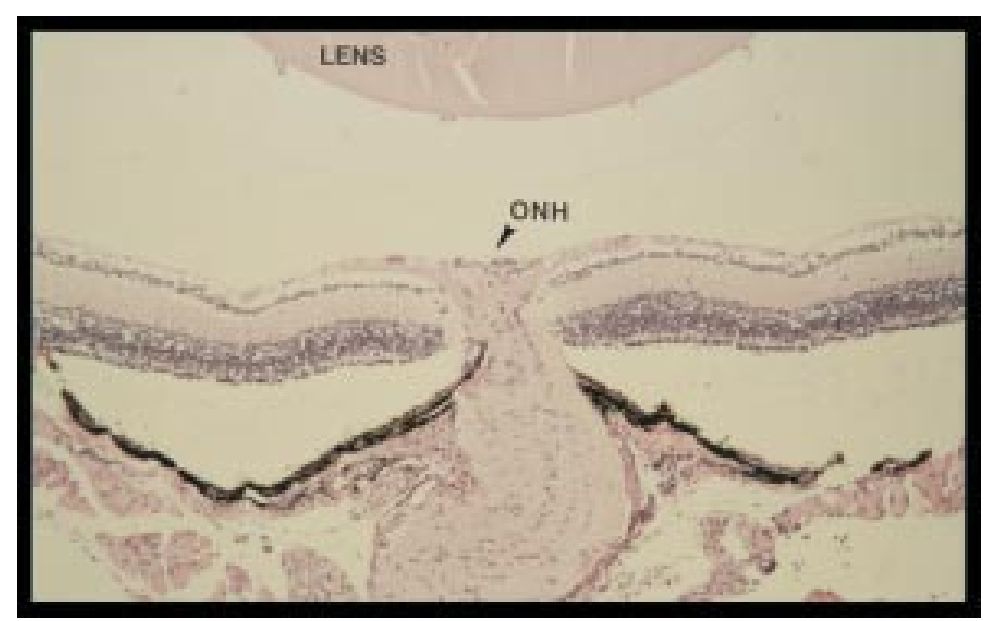

Figure 1 Eyes were sectioned to the level of optic nerve head (ONH) and only sections taken close to either side of the ONH were evaluated to ensure that retinas of normally equivalent thicknesses were compared. A 5 um paraffin wax section counterstained with haematoxylin and eosin (10x objective) taken from 22 day old $r d$ mouse 10 days after intravitreal injection of $1 \mu l$ of $P B S$. $\mu \mathrm{g}$ CD4 (YTS 191) and/or CD8 (Yts 169) specific monoclonal antibodies ${ }^{20} 1$ day before intravitreal injection.

\section{HISTOLOGY}

Animals were sacrificed 10 and 20 days post-injection. Eyes were removed, fixed in $10 \%$ neutral buffered formalin, embedded in paraffin wax using a routine protocol, and sectioned to the level of optic nerve head $(\mathrm{ONH})$. Only sections taken close to either side of the $\mathrm{ONH}$ were evaluated to ensure that areas of retinas of normally equivalent thicknesses were compared. The $5 \mu \mathrm{m}$ sections were counterstained with haematoxylin and eosin. Two independent observers assessed the number of rows of photoreceptor nuclei in the outer nuclear layer (ONL) of several sections from each eye. The scoring system was as follows: 0 , a sparse row of photoreceptors; 1 , a single row of photoreceptors; 2 , between one and two rows of photoreceptors; 3 , between two and three rows of photoreceptors, and so on. For animals sacrificed 10 days post-injection, between six and 12 eyes were analysed per experimental group while for animals sacrificed 20 days post-injection, between 10 and 20 eyes were analysed. The standard error of the mean ONL score were calculated and a non-paired, two tailed Mann-Whitney U test was used to calculate significance.

\section{Results}

Litters of 12 day old $r d$ mice were injected intravitreally with AV.LacZ. The injections were performed intravitreally rather than subretinally since subretinal injection results in a partial detachment with localised transduction and in subsequent analysis it would be more difficult to compare equivalent areas. Twelve day old animals were chosen because the eyes are large enough to allow injections of consistent quality. In very young mice it is difficult to inject the eyes without causing damage to the lens. In order to control for slight differences in age of animals, litters of $r d$ mice were divided randomly between the different experimental groups. Animals were injected with AV.LacZ, of which a proportion were depleted of either CD4+ or CD8+ $\mathrm{T}$ cells or both. Control animals were injected with PBS. The mice were sacrificed after 10 or 20 days postinjection and the eyes embedded in paraffin wax and sectioned. Only sections taken close to either side of the optic nerve head were evaluated to enable us to compare retinas of normally equivalent thicknesses (Fig 1). The outer nuclear layer (ONL) of the retina contains photoreceptor cell nuclei. At 22 days in a normal mouse retina the ONL is $8-10$ rows thick whereas in the $r d$ mouse the ONL is reduced to one row. In 22 day old $r d$ mice, 10 days after intravitreal injection of AV.LacZ, the ONL contains an average of 2.5 rows compared with 1.5 in PBS injected animals (Figs 2 and 3), p<0.005. The protective effect of AV.LacZ is negated by immune suppression. In animals injected with AV.LacZ and depleted of both CD4+ and CD8+ T cells there was no statistically significant difference between the 


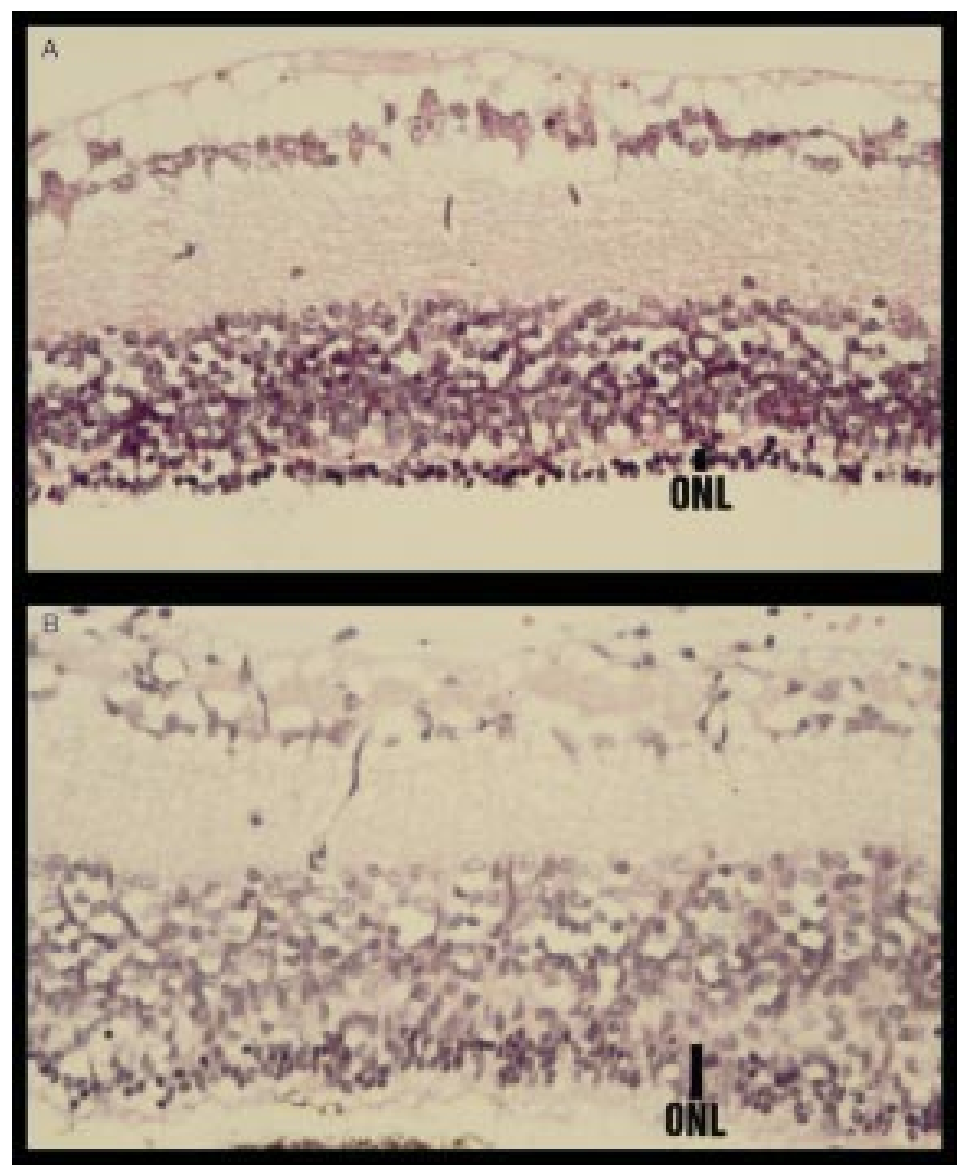

Figure 2 Examples of sections taken close to the $O N H$ region of 22 day old rd mice 10 days after intravitreal injection of either (A) $1 \mu \mathrm{l}$ of PBS or (B) $1 \mu \mathrm{l}$ of AV.LacZ (1×10 pfu). The $5 \mu \mathrm{m}$ paraffin sections were counterstained with haematoxylin and eosin (40X objective). Eyes injected with PBS had between one and two rows of photoreceptors in the outer nuclear layer (ONL) whereas in some eyes injected with AV.Lac $Z$ there were between three and four rows of photoreceptors.

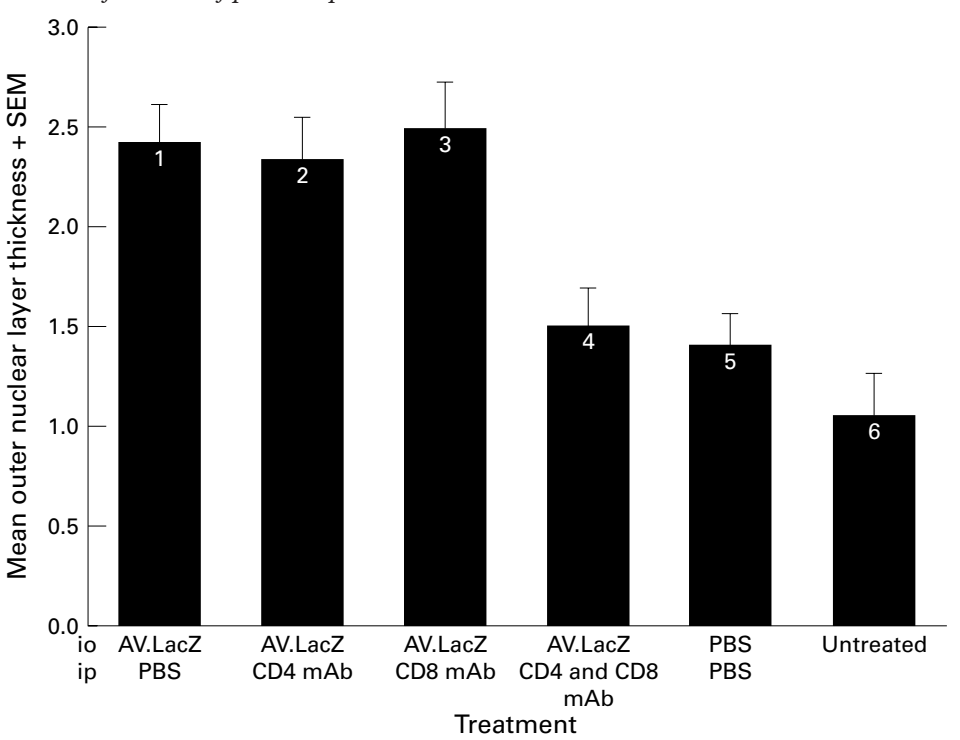

Figure 3 Comparison of $r$ ONL thickness in six groups of animals following intraocular injection (io) of either PBS or AV.LacZ with and without T cell ablation as a result of intraperitoneal injection (ip) of either PBS or monoclonal antibodies ( $m A \mathrm{~b})$ specific for $C D 4$ or CD8. The number of eyes in each treatment group (from 1 to 6 ) is 12, 6, 6, 8, 10, and 10 respectively. The scoring system was as follows: 0, a sparse row of photoreceptors; 1 , a single row of photoreceptors; 2, between one and two rows of photoreceptors; 3, between two and three rows of photoreceptors, and so on. The data show the standard error of the mean ONL score. The difference in ONL thickness between treatment groups 1, 2, and 3 is not statistically significant. There are also no statistically significant differences in ONL thickness between treatment groups 4, 5, and 6. There is, however, a significant difference between groups 1 and $4(p>0.01) ; 1$ and $5(p<0.005) ; 1$ and $6(p<0.001) ; 2$ and $4(p<0.05) ; 2$ and 5 $(p<0.05) ; 2$ and $6(p<0.005) ; 3$ and $4(p<0.05) ; 3$ and $5(p<0.05) ; 3$ and $6(p<0.005)$.
ONL thickness compared with animals injected with only PBS. Depletion of just one type of these $\mathrm{T}$ cells did not alter the effect of AV.LacZ. This is consistent with our previous observation that depletion of both CD4 and CD8 cells was required for suppression of immune responses following ocular AV administration. ${ }^{18}$ Twenty days after injection, the ONL was slightly thicker in AV.LacZ injected animals compared with controls. However, the differences were not statistically significant (data not shown).

\section{Discussion}

Immune responses following intravitreal and subretinal administration of adenoviral vectors have been reported previously. In this study we have demonstrated that a $\mathrm{T}$ cell mediated immune response is able to alter the course of retinal degeneration in the $r d$ mouse. Earlier studies have shown that sham PBS injections are able to slow degeneration in the $r d y$ rat retinal degeneration. ${ }^{21}$ This phenomenon appears to be due to stimulation of growth factors which promote photoreceptor survival. Although it has been shown in this and other studies $^{22}$ that intraocular PBS injections do not have a significant effect on photoreceptor survival in the $r d$ mouse, we have demonstrated here that adenoviral administration does protect photoreceptors. This appears to be due specifically to the host immune response. Activated $\mathrm{T}$ cells have been shown to produce brain derived neurotrophic factor (BDNF) in vitro and in the $\mathrm{CNS}^{23}$ and autoimmune $\mathrm{T}$ cells have been shown to protect axtomised neurons. ${ }^{24}$ Inflammation in the eye may also result in upregulation of neurotrophic factors. Confirmation of this and the identification of particular factors requires further study.

A number of groups have reported short term delay in loss of photoreceptor cells in the rd mouse following AV mediated gene transfer of a variety of genes but the mechanisms of the delay have not yet been proved. Here we also report a short term delay in the loss of photoreceptor cells but show that the mechanism is that of an immune response. Differences in the types and titres of recombinant adenovirus used, variation in injection technique and the genetic background of animals, the use of different promoters (and thus expression levels), and differences in the method of morphological assessment make it difficult to compare the results presented here with other studies involving AV.LacZ administration to the $r d$ mouse eye. However, in at least one other system, intraocular AV.LacZ administration has resulted in a neuroprotective effect. ${ }^{25}$ While in some studies it appears that administration of a vector carrying a reporter gene is less effective than vectors carrying functional constructs, differences in immunogenicity may in part be responsible for this. For instance, secreted gene products which contain a Myc tag have been delivered ${ }^{15}$ and these may be more immunogenic than an intracellular reporter gene product. It would be interesting to determine the relative importance of the immune response to transgene and viral gene 
products. In order to exclude the possibility that an immune response to vector and/or transgene product is responsible for delaying loss of photoreceptors in this mouse model it is important to use controls with immunosuppression or an inactive "functional" gene. Where the target cells are photoreceptors, which are transduced by AV with an efficiency of $2-3 \%$, it is particularly important to demonstrate the mechanism of rescue.

1 Bird AC. Retinal photoreceptor dystrophies. Am f Ophthalmol 1995;119:543-62.

2 Dryja TP, McGee TL, Reichel E, et al. A point mutation of rhodopsin gene in one form of retinitis pigmentosa. Nature 1990;343:364-6.

3 Farrar GJ, Kenna P, Jordan SA, et al. A three base-pair deletion in the peripherin-RDS gene in one form of retinitis tion in the peripherin-RDS gene in

pigmentosa. Nature $1991 ; 354: 478-80$.
4 McLaughlin ME, Sandberg MA, Berson EL, et al. Recessive mutations in the gene encoding the $\beta$-subunit of rod phosmutations in the gene encoding the $\beta$-subunit of rod phosphodiesterase in patien

5 Dryja TP, Finn JT, Peng Y-W, et al. Mutations in the gene encoding the $\alpha$ subunit of the rod cGMP-gated channel in autosomal recessive retinitis pigmentosa. Proc Natl Acad Sci USA 1995;92:10177-81.

6 Bascom RA, Liu L, Heckenlively JR, et al. Mutation analysis of the ROM1 gene in retinitis pigmentosa. Hum Mol Genet 1995;4:1895-902.

7 Freund CL, Gregory-Evans CY, Furukawa T, et al. Cone-rod dystrophy due to mutations in a novel photoreceptor-specific homeobox gene (CRX) essential for maintenance of the photoreceptor. Cell 1997;91:543-53.

8 Bessant AR, Payne AM, Mitton KP, et al. A mutation in NRL is associated with autosomal dominant retinitis pigmentosa. Nat Genet 1999;21:355-6.

9 Farber DB, Flannery JG, Bowes-Rickman C. The $r d$ mouse story: seventy years of research on an animal model of story: seventy years of research on an animal model of
inherited retinal degeneration. Prog Ret Eye Res 1994;13: inherited.

10 Portera-Cailliau C, Sung CH, Nathans J, et al. Apoptotic photoreceptor cell death in mouse models of retinitis pigmentosa. Proc Natl Acad Sci USA 1994;91:974-8.

11 Bennett J, Wilson J, Sun D, et al. Adenovirus vectormediated in vivo gene transfer into adult murine retina. Invest Ophthalmol Vis Sci 1994;35:2535-42.
12 Jomary C, Grist J, Neal MJ, et al. Rescue of photoreceptor function by AAV-mediated gene transfer in a mouse model of inherited retinal degeneration. Gene Therapy 1997;4: 683-90.

13 Kumar-Singh R, Farber DB. Encapsidated adenovirus mini-chromosome-mediated delivery of genes to the retina: application to the rescue of photoreceptor degeneration. Hum Mol Genet 1998;7:1893-900.

14 Bennett J, Zeng Y, Bajwa R, et al. Adenovirus-mediated delivery of rhodopsin-promoted bcl-2 results in a delay in photoreceptor cell death in $\mathrm{rd} / \mathrm{rd}$ mouse. Gene Ther 1998;5: 1156-64.

15 Cayouette M, Gravel C. Adenovirus-mediated gene transfer of ciliary neurotrophic factor can prevent photoreceptor degeneration in the retinal degeneration (rd) mouse. Hum Gene Ther 1997;8:423-30.

16 MohandSaid S, DeudonCombe A, Hicks D, et al. Normal retina releases a diffusible factor stimulating cone survival in the retinal degeneration mouse. Proc Natl Acad Sci USA 1998;95:8357-62.

17 Mohandsaid S, Hicks D, Simonutti M, et al. Photoreceptor transplants increase host cone survival in the retinal degeneration (rd) mouse. Ophthalmic Res 1997;29:290-7.

18 Reichel MB, Ali RR, Thrasher AJ, et al. Immune responses limit adenovirally-mediated gene expression in the adult mouse eye. Gene Ther 1998;5:1038-46.

19 Byrnes AP, Rusby JE, Wood MJA, et al. Adenovirus gene ransfer causes inflammation in the brain. Neuroscience 1995;66:1015-24.

20 O'Neill JK, Baker D, Davison AN, et al. Control of immune-mediated disease of the central nervous system with monoclonal (CD4-specific) antibodies. 7 Neuroimmunol 1993;45:1-14.

21 Wen R, Song $\mathrm{Y}$, Cheng $\mathrm{T}$, et al. Injury-induced up-regulation of BFGF and CNTF messenger-RNAs in the rat retina. 7 Neurosci 1995;15:7377-85.

22 Cao W, Wen R, Li F, et al. Mechanical injury increases bFGF and CNTF mRMA expression in the mouse retina. Exp Eye Res 1997;65:241-8.

23 Kerschensteiner M, Gallmeier E, Behrens L, et al. Activated human $\mathrm{T}$ cells, $\mathrm{B}$ cells, and monocytes produce brainderived neurotrophic factor in vitro and in inflammatory brain lesions: a neuroprotective role of inflammation? $\mathcal{F}$ Exp Med 1999;189:865-70.

24 Moalem G, Leibowitz Amit R, Yoles E, et al. Autoimmune T cells protect neurons from secondary degeneration after central nervous system axotomy. Nat Med 1999;5:49-55.

25 DiPolo A, Aigner LJ, Dunn RJ, et al. Prolonged delivery of brain-derived neurotrophic factor by adenovirus-infected Muller cells temporarily rescues injured retinal ganglion cells. Proc Natl Acad Sci USA 1998;95:3978-83. 